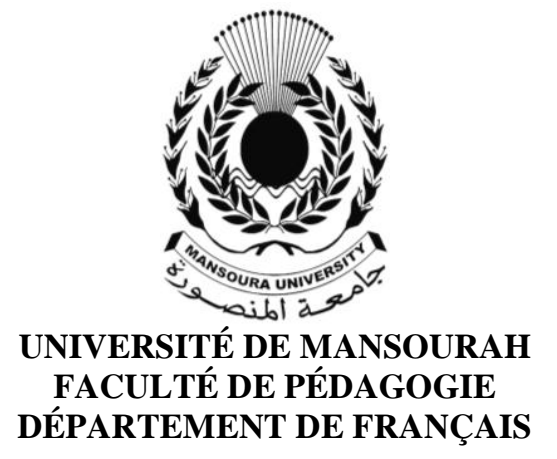

\title{
LES ASPECTS DE L'ASSIMILATION PHONOLOGIQUE EN FRANÇAIS ET EN ARABE (ÉTUDE CONTRASTIVE)
}

\section{By \\ Dr. ABDELWAHAB ELSAADANI}

Professeur adjoint en Linguistique française

Faculté de Pédagogie

Université de Mansourah

Revue de la faculté des lettres de Mansourah

$54^{\text {Th }}$ ISSUE- Jan. 2014 


\section{LES ASPECTS DE L'ASSIMILATION PHONOLOGIQUE EN FRANÇAIS ET EN ARABE (ÉTUDE CONTRASTIVE) Dr. Abdelwahab Elsaadani}

\section{Introduction}

Dans la chaîne parlée, les sons ne se réalisent pas toujours sous leur forme prototypique; ils peuvent se manifester de façons très variables pour diverses raisons dont les plus importantes sont les propriétés articulatoires d'un son par rapport à ceux qui l'entourent (qui le précèdent et le suivent). Autrement dit, les deux sons en contact, de type articulatoire différent, tendent à se rapprocher, à s'assimiler le plus possible sans toutefois porter atteinte ni à la compréhension ni à la forme graphique. Une phrase comme « je ne sais pas » peut être prononcée de différentes façons, comme

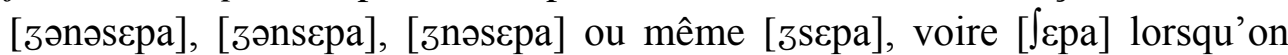
enchaîne les mots rapidement. On voit que l'image acoustique des monèmes change selon le moment où ils sont dits isolés ou prononcés enchaînés comme dans [3ə ou J]. Cette source de variation peut modifier profondément les caractéristiques acoustiques d'un son. Mais les variations obéissent aux règles phonologiques spécifiques à la langue en question. En français par exemple, le mot français terminé par une consonne occlusive non voisée comme «frites» est prononcé [frid] quand il est suivi d' un mot ayant une consonne initiale voisée (ex., Les frites belges).

En arabe, au contact d'une sonore, une sourde devient sonore ou inversement. Ainsi le verbe (عدت) [Gud+ta], se prononce couramment / Gutta / avec l'assimilation du [d/د] voisé du verbe au [t/ت] du suffixe et peut s'écrire soit (عدت) qui est parfaitement clair, soit (عتّ) qui est plus obscur. C'est pourquoi, «l'arabe moderne a tendance à préférer, dans ce type de cas, la première orthographe, qui préserve la clarté morphologique du mot.»1

Ce phénomène illustré dans nos exemples est l'assimilation phonique qui est un type très fréquent de variation phonologique subie par un son au contact d'un son voisin et qui tend à réduire les différences entre les deux sons: l'un des deux sons acquiert une ou plusieurs caractéristiques propres au son voisin. À l'inverse, lorsque deux sons semblables, en contact plus ou moins direct, s'éloignent l'un de l'autre, on parle de la dissimilation.

1 - Djamel Kouloughli, Grammaire de l'arabe d'aujourd'hui, Paris, Pocket-Langues pour tous, 1994, p.24. 


\section{Objectif de l'étude}

L'étude a pour but d'examiner et de décrire le phénomène de l'assimilation comme un aspect de variation phonologique qui dépend à la fois des traits pertinents et de la position des phonèmes en contexte phonique. On tente de savoir comment et par quels mécanismes, le degré et le type d'assimilation dépendent du contexte des phonèmes.

\section{La méthode}

Notre description phonologique de l'assimilation en français et en arabe se déroulera dans le cadre de l'approche fonctionnaliste où «la phonologie est une phonétique fonctionnelle et structurale» ${ }^{2}$ et selon laquelle l'analyse phonologique suit deux étapes principales: au premier temps «on identifie les traits pertinents qui différencient les unités distinctives les unes des autres afin de définir chacune d'entre elles par les traits pertinents qui la caractérisent. » Au second temps, «on étudie la manière dont les unités distinctives se combinent pour donner chair et forme au signifiant des unités

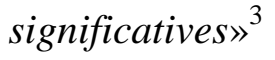

\section{Corpus de l'étude}

Les exemples cités dans l'étude en langue française sont tirés du français parlé, alors que ceux cités en langue arabe sont à la fois empruntés de l'arabe moderne et du Saint Coran.

\section{Définition de l'assimilation}

L'assimilation est à la fois un phénomène très ancien et très jeune; elle a été précieusement étudiée par les grammairiens arabes anciens. Dans son chef-d'œuvre" al-Kitāb "*, le prestigieux grammairien du huitième siècle Sibawayh $(\mathrm{m} .180 / 796)^{*}$, a décrit l'assimilation dans plusieurs positions ، il l'a nommée «Palmudarą المضارعة ${ }^{4}$ c'est-à-dire (l'assimilation), «Paltaqrib التقريب (le rapprochement) et «?alidgam

2 -André Martinet, La Description Phonologique avec application au parler franco provençal d'Hauteville (Savoie), Genève-Paris, 1956, p. 15.

3 - Builles (Jean Michel), Manuel de linguistique Descriptive, Le point de vue fonctionnaliste, Paris, Nathan, 1998.P.187.

* - Al-Kitāb de Sibawayh a été traduit en français en deux volumes à Paris entre (18811889) par l'orientaliste Hartwig Derenbourg (1844-1908).Malheureusement, je n'ai pas pu trouver cette traduction.

* -Les dates citées après les noms des personnages de la tradition arabes reportent, après la lettre m., la date du décès selon le calendrier de l'Hégire, suivie de la date correspondante selon le calendrier chrétien.

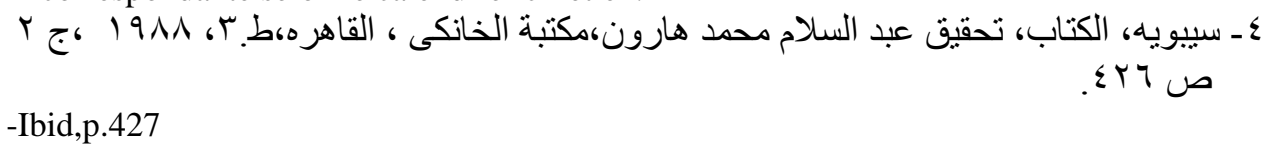


إخاد الادغام (la gémination). Dans son livre (al-khașā'is /Les Particularités الخصائص (bn Jinni, (m.393/1002) a traité le phénomène de l'assimilation dans un chapitre intitulé (?alidgam ralasgar الادغام الاصغر / la petite gémination) et l'a défini comme* "un rapprochement des traits pertinents d'un son à ceux d'un autre son voisin sans faire une gémination.» ${ }^{7}$

En général, les phonologues modernes définissent l'assimilation comme «les différentes sortes de changements dont un son est susceptibles d'être affecté quand il subit l'influence d'un son voisin: deux sons contigus tendent à acquérir un ou plusieurs caractères communs $»^{8}$, autrement dit, «c'est la tendance de deux phonèmes différents contigus à se ressembler ou à acquérir un ou plusieurs traits communs partiellement ou totalement. » 9 . Selon le (ou les) traits articulatoires que le phonème peut acquérir, l'assimilation peut être donc «partielle lorsque le phonème assimilé garde un ou plusieurs traits distinctifs, totale lorsqu'il adopte entièrement ceux du phonème contigu et perd les siens». ${ }^{10}$ Ainsi «je t'attends», peut être prononcé [zatatã] ou, après la chute[ə], [క̌tatã] lorsque l'assimilation est partielle, [Jtatã] lorsqu'elle est totale. »11 Au premier temps, la consonne [t] transfère partiellement le trait [+ sourd] au [3], qu'on prononce[3̌] ainsi plus proche de []]; au deuxième temps, le [3] a totalement perdu son trait [+ sonore], changeant en [j], sous l'effet du sourd [t].

L'assimilation réfère donc à la propagation d'un trait distinctif d'un son $\mathrm{X}$ en contexte sur le son $\mathrm{Y}$. Ce phénomène se produit donc au cours de la rencontre de deux consonnes dont la plus forte assimile la plus douce (i.e.la plus faible) en lui donnant certains de ses caractères. ${ }^{12}$

6- Ibid., p.404

* - Les citations arabes sont traduites par le chercheur.

V- ألا دغام الاصغر عند ابن جنى هو 》 ثقريب الحرف من الحرف وادنـاؤه من غير ادغام يكون

هنـاك • « ابن جنـى ،الخصـائص ،تحقيق:محمد على النجار، دار الكتب المصسرية،ج .ب،

$.910190 \mathrm{~V}$

8 - Carton (Fernand), Introduction à la phonétique française, Paris, Dunod, 1997, P.82.

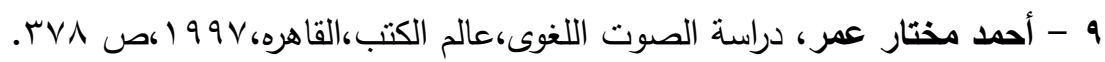

10 - Mounin (Georges), Dictionnaire de la linguistique, Paris, PUF, 1995, P.43

* - On utilise les signes diacritiques pour indiquer une sonorité ou un assourdissement qui ne sont pas propres au son en question: v pour voisement, $v$ renversé pour dévoisement sur ou sous la consonne.

11 -Derivery (Nicole), La phonétique du français, Paris, Seuil, 1997, P.38.

${ }^{12}$ - Selon Bertil Malmberg, il y a des consonnes fortes et des consonnes douces. En français, les occlusives sourdes/p, $\boldsymbol{t}$ et $\mathrm{k} /$ et les spirantes $/ \boldsymbol{f}, \boldsymbol{s}$ et $\mathrm{J} / \mathrm{sont}$ des fortes, toutes les autres 


\section{Les contraintes de l'assimilation}

L'assimilation varie selon deux critères:

- Les traits pertinents qui différencient les phonèmes les uns des autres;

- La position des consonnes en contact: dans la même syllabe ou dans deux syllabes différentes.

\subsection{Les traits pertinents}

A la suite d'André Martinet, le trait pertinent est " un ensemble de caractéristiques phoniques distinctives qui ne se trouvent dissociées nulle part dans le système ${ }^{13}{ }_{\gg}$. C'est donc un trait simple ou complexe qui permet à tout seul de distinguer deux phonèmes. Les phonèmes sont donc organisés dans un système, dans la mesure où ils s'opposent les uns aux autres par des traits qui les composent. En français et en arabe, le voisement, le lieu d'articulation, le mode d'articulation, la nasalité, la vibration et le trait latéral $^{14}$ constituent les corrélations $^{*}$ permettant de décrire tous les phonèmes.

\section{A. Le voisement}

Les cordes vocales sont les responsables de la production du son fondamental de la voix. Le mot voix désigne l'ensemble des sons produits par les vibrations des cordes vocales. Les sons dont la production ne s'accompagne pas de la vibration des cordes vocales sont dits non voisés ou sourds. Les voyelles sont toujours voisées, alors qu'il existe des consonnes voisées et d'autres non voisées. Le voisement et le dévoisement constituent dans beaucoup de langues des traits phonologiquement pertinents. En français, il existe une série de six consonnes sourdes qui s'oppose à une série de six consonnes sonores situées aux mêmes points d'articulation que les sourdes (voir tableau 1): le/b/ de bon est une bilabiale située au même point d'articulation que le /p/ de pont, la seule différence entre les deux

des douces. Parmi les occlusives et les spirantes, il y a deux séries, une série des fortes qui s'opposent à la série des douces ( $\boldsymbol{p}$ à $\boldsymbol{b}, \boldsymbol{s}$ à $\boldsymbol{z}$, etc.). Les nasales et les liquides sont toujours des douces, de même que les semi-voyelles.

(Bertil Malmberg, La Phonétique, Paris, PUF, 4 éd. 1987, P.60.)

13 - André Martinet, "Substance phonique et traits pertinents", in La Linguistique synchronique, Paris, PUF, 1970, p. 144.

14 - Choi-Jonin (Injoo) \& Delhy (Corinne), Introduction à la méthodologie en linguistique, Paris, Presses universitaires de Strasbourg, 1998.pp.25-29.

* - On appelle "corrélation" un ensemble des couples de phonèmes dont les membres se différencient par un même trait pertinent. En français, les couples /p/-/b/, /f/-/v/, /t/-/d/, /s/-/z/, / / /-/3/ et /k/-/g/ forment une corrélation. ( Builles (Jean Michel), Manuel de linguistique Descriptive, Op.cit., 
consonnes réside dans la sonorité. ${ }^{15}$ Ces paires de phonèmes que Martinet appelle phonèmes corrélatifs ${ }^{16}$ peuvent être illustrés dans le tableau suivant:

\begin{tabular}{|l|l|l|l|l|l|l|}
\hline «voisés» & $/ \mathrm{b} /$ & $/ \mathrm{v} /$ & $/ \mathrm{d} /$ & $/ \mathrm{z} /$ & $/ \mathrm{z} /$ & $/ \mathrm{g} /$ \\
\hline «non voisés» & $/ \mathrm{p} /$ & $/ \mathrm{f} /$ & $/ \mathrm{t} /$ & $/ \mathrm{s} /$ & $/ \mathrm{J} /$ & $/ \mathrm{k} /$ \\
\hline
\end{tabular}

Tableau 1

D'après Sibawayh ${ }^{17}$, les consonnes arabes sont classifiées en dix-neuf voisés (mağhūrs) et dix sourdes (mahmūs):

\begin{tabular}{|c|c|}
\hline Les voisés (almağhūrs) & 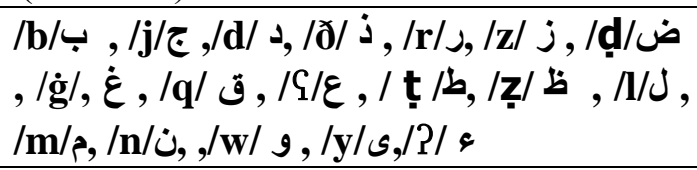 \\
\hline $\begin{array}{l}\text { Les non } \\
\text { (almahmūs) }\end{array}$ & 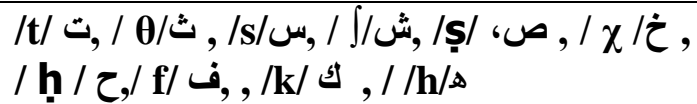 \\
\hline
\end{tabular}

\section{B. Le mode d'articulation}

A l'opposé des voyelles dont la production se distingue par l'absence d'obstruction au passage de l'air dans la cavité buccale, la production des consonnes nécessite la fermeture totale ou partielle en un endroit quelconque de la cavité orale ou nasale. La fermeture totale produit les consonnes appelées occlusives; tandis qu'on parle de consonnes constrictives lorsqu'il y a une fermeture partielle à un endroit quelconque de la cavité buccale.

\section{Le lieu d'articulation}

Si le lieu d'articulation des voyelles se définit par la position de la langue (antérieure ou postérieure), l'articulation d'une consonne est déterminée non seulement par le voisement et le mode d'articulation mais aussi par le lieu d'articulation, à savoir l'endroit où a lieu l'obstruction totale ou partielle du passage de l'air. Considérons les phonèmes /d / / et / $/$ / $/$ / dans $(/ \mathrm{du} / \sim / \mathrm{fu} /$,

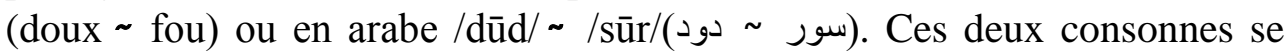
distinguent par deux traits distinctifs: /d/ est une occlusive voisée (mode d'articulation), dentale (lieu d'articulation), tandis que /f/ est une constrictive non voisée (mode d'articulation), labiodentale (lieu d'articulation).

15 - Walter, (Henriette), La phonologie du français, Paris, PUF, 4e éd., 1977, P.28.

16 - Martinet, (André), La phonologie, in La linguistique, Les introuvables d'André Martinet, volume36, 2001-1/2, Paris, PUF, 2001, P.262.

IV 


\section{La nasalité}

L'articulation orale est l'articulation la plus fréquente dans les sons d'une langue lorsque le voile du palais est relevé en fermant le passage aux fosses nasales. Si le voile du palais est au contraire abaissé, l'air passe alors à la fois par la cavité nasale, utilisé comme résonateur supplémentaire, en plus par la cavité buccal. Les sons produits sont alors nasalisés. En français, la production des voyelles nasales $([\tilde{\varepsilon}][\tilde{œ}][\tilde{o}][\tilde{a}])$ s'effectue sans aucune obstruction au passage de l'air par la cavité nasale, alors que celle des consonnes nasales ([m] [n] [n]) s'effectue toujours par le passage de l'air par la cavité nasale mais accompagné d'une obstruction à un endroit de la cavité buccale. Quant à l'arabe, le mécanisme phonologique est presque le même. $\mathrm{Au}$ cours de la production des nasales les deux cavités orale et nasale sont donc normalement utilisées. En arabe, il y deux consonnes nasales (ghounna غنة c'est-à-dire un nasillement ou nasalisation) le /m/ et le /n/.

\section{E. Les deux liquides: la latérale [I] et la vibrante [r]}

En français, la consonne alvéolaire voisée [1] a un seul trait qui la distingue de tous les autres phonèmes: c'est la seule latérale. Un seul trait suffit aussi à distinguer [r] : c'est la seule vibrante en français. Les deux consonnes [1] et [r] sont des sonantes.

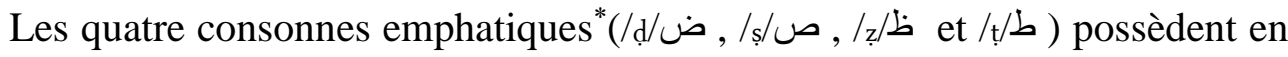
effet des correspondants non emphatiques en arabe standard moderne. Le trait pertinent d'emphase, distinguant les quatre unités consonantiques, peut être appliqué également sur la latérale dentale voisée /1/ ; elle peut être considérée comme " une consonne emphatique latérale postdentale /l/ qui se produit dans un environnement extrêmement limité et seulement devant/al et /aa/. Dans le langage littéraire, on le trouve dans le mot/Allah/ (Dieu) et ses dérivés mais dans les dialectes il est plus commun. ${ }^{18}$

\section{L'assimilation du /l/ (Lām) de l'article défini}

Le Lām(J) de l'article défini / $/ \mathrm{Ral} /$ est assimilé par la première consonne du nom qui le suit et dont le point d'articulation est voisin du sien, cette proximité empêche que l'on prononce distinctement (à une vitesse normale

* - Selon Troubetzkoy les phonèmes porteurs de la marque de vélarisation emphatique صadmettent la corrélation avec des phonèmes non emphatiques. En plus du -, , ל حl'emphatique $/ \mathrm{h} / \mathrm{s}$ et considère $\chi / \dot{\tau}$ et $/ \mathrm{S} / \varepsilon$ comme des emphatiques. (Troubetzkoy, Principes de phonologie, Klincksiek, Paris, 1970. p.148.)

18 - DJOUDI ( Mahieddin), Contribution à l'étude et à la reconnaissance automatique de la parole en Arabe standard, thèse de Doctorat de l'université de Nancy 1, Novembre 1991, p.48. 
d'élocution) chacune des deux lettres, c.-à-d. le Lām puis la lettre suivante. Dans ce cas :

- Au niveau phonétique, le Lām n'est plus prononcé et lui est substituée la lettre suivante qui se trouve ainsi prononcée deux fois : une fois à la place du Lām + une fois pour elle-même.

- Au niveau graphique par contre, le Lām n'est jamais affecté et est donc toujours écrit. Une $\mathbf{C h a d d a}^{19}$ grammaticale [] vient marquer le redoublement de la consonne voisine.

Les lettres assimilantes, appelées les lettres solaires (حُروف شمسيّة), sont au

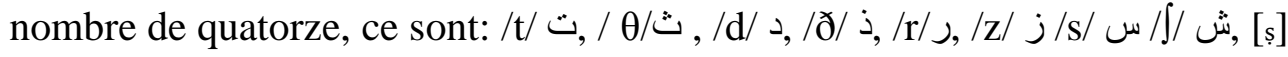
(ن' C'est ainsi que "Au lieu de/allams/ (soleil) on prononce /a Jams/. La consonne/l/ de l'article est assimilée dans la prononciation par la consonne assimilante qui la suit c'est-à-dire $/ \mathrm{J} . » 20$ comme dans /at' tūt/النّّت, /an' nās/ النّاس/.

Les consonnes non assimilantes sont dites lettres lunaires (حُروف قمريّة) parce que deux d'entre elles se trouvent dans le substantif (قمر / lune) servi comme exemple de non assimilante /alqamar/. Comme dans /al-bāb/البابر

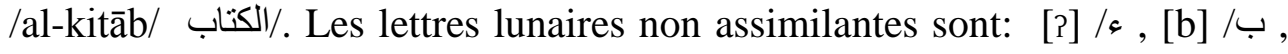
$[\chi] / \dot{\tau},[\mathrm{h}] / \mathrm{\tau},[3] / \mathrm{C},[\dot{\mathrm{g}}] /, \dot{\varepsilon},[\mathrm{c}] / \mathcal{\varepsilon},[\mathrm{q}] / \mathrm{G},[\mathrm{k}] / \mathrm{s},[\mathrm{m}] / \mathrm{\rho},[\mathrm{h}] / \mathrm{s},[\mathrm{w}] / \mathrm{g}$, [y]/ $/$ e.

Appliquons les règles de l'assimilation du Lām sur les versets 3 et 4 de la

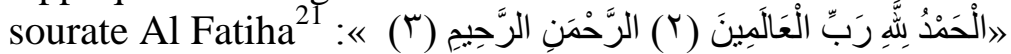

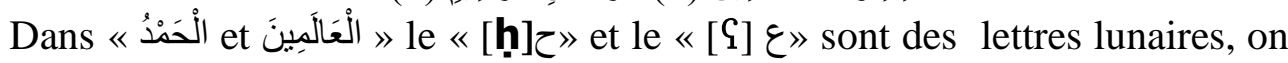

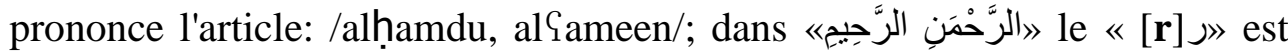
une lettre solaire, les deux articles ne sont pas prononcés et le «J» est renforcé: /arraḥmani,arraḥeemi/.

Après avoir traité les traits pertinents des phonèmes dans les deux langues, on regroupe, dans le tableau $3^{22}$, les traits pertinents des consonnes françaises selon les six corrélations citées plus haut :

19 -La Chadda [̈] est un graphème (symbole sous forme du chiffre 3 couché de dos) que l'on place au-dessus d'une consonne redoublée: « il marque que la consonne qui le porte est géminée et compte donc deux.Ainsi le mot [qițt] chat s'écrit قُ avec un seul țā surmonté d'une chadda. "

Djamel Kouloughli, Op.cit, pp.12-13.

20 - DJOUDI ( Mahieddin), Op.cit. p.62.

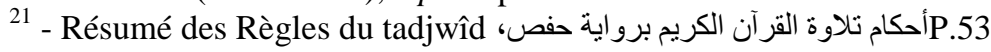

www.islamhouse.com/d/files/fr/ih.../fr-Islamhouse-regles_tajwid.pdf 


\begin{tabular}{|c|c|c|c|c|c|c|c|c|c|}
\hline \multicolumn{3}{|c|}{ Lieu d'articulation } & \multirow{2}{*}{$\begin{array}{c}\text { bilabial } \\
{[\mathbf{p}]} \\
\end{array}$} & \multirow{2}{*}{$\begin{array}{l}\text { labio- } \\
\text { dental }\end{array}$} & \multirow{2}{*}{$\begin{array}{c}\begin{array}{r}\text { Apico- } \\
\text { alvéolaire } \\
\text { ou apico- } \\
\text { dental }\end{array} \\
{[\mathbf{t}]}\end{array}$} & \multirow{2}{*}{$\begin{array}{l}\text { Pré-dorso- } \\
\text { alvéolaire }\end{array}$} & \multirow{2}{*}{$\begin{array}{c}\text { Apico- } \\
\text { (pré)palatal }\end{array}$} & \multirow{2}{*}{$\begin{array}{c}\begin{array}{c}\text { Dorso- } \\
\text { palatal ou } \\
\text { dorso-vélaire }\end{array} \\
{[\mathbf{k}]}\end{array}$} & \multirow{2}{*}{$\begin{array}{l}\text { Dorso- } \\
\text { uvulaire }\end{array}$} \\
\hline$y$ & orales & \begin{tabular}{|c|} 
non \\
voisées
\end{tabular} & & & & & & & \\
\hline 产 & & voisées & [b] & & [d] & & & [g] & \\
\hline ठ & nasales & & {$[\mathbf{m}]$} & & [n] & & & [n] & \\
\hline \multirow{2}{*}{\multicolumn{2}{|c|}{ fricatives }} & $\begin{array}{c}\text { non } \\
\text { voisées }\end{array}$ & & [f] & & [s] & [J] & & \\
\hline & & voisées & & {$[\mathbf{v}]$} & & {$[\mathbf{z}]$} & [3] & & \\
\hline \multicolumn{10}{|c|}{ latériales } \\
\hline & orantes & & & & [1] & & & & {$[\mathbf{R}]$} \\
\hline
\end{tabular}

\section{Tableau 3}

En plus des traits pertinents communs avec les phonèmes du français, le système phonétique de l'arabe tire son originalité de la présence des

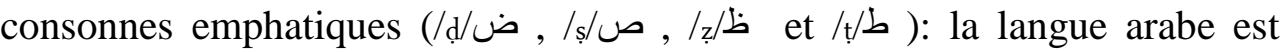
souvent appelée la langue du /ḍad/; un phonème qui n'existe qu'en arabe et qui est d'ailleurs difficile à prononcer.

Dans le tableau 4, on regroupe les traits pertinents des consonnes arabes:

\begin{tabular}{|c|c|c|c|c|c|c|}
\hline $\begin{array}{l}\text { Modes } \\
\text { Lieüx }\end{array}$ & occlusives & Emphatiques & Fricatives & Nasales & Liquides & Semi-voyelles \\
\hline Labiales & ب[ [b] & & ف] [f] & $\rho /[\mathrm{m}]$ & & $9 /[w]$ \\
\hline Interdentales & & b/[z] & \begin{tabular}{cl}
$\dot{j}$ & \multirow{2}{*}{} \\
{$[\tilde{\theta}]$} & {$[\theta]$}
\end{tabular} & & & \\
\hline Dentales & $\begin{array}{cc}\vec{J} & د \\
\text { [d] } & {[\mathrm{t}]} \\
\end{array}$ & $\begin{array}{cl}\dot{\omega} & b \\
{[d]} & {[t]}\end{array}$ & & ن/[n] & $\begin{array}{ll}j & j \\
{[1]} & {[r]}\end{array}$ & \\
\hline Sifflantes & & (ș] / & $\begin{array}{ll}j & w \\
{[z]} & {[s]}\end{array}$ & & & \\
\hline Palatales & $\overline{e /[j]}$ & & 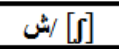 & & & $\mathrm{s/[ \mathbf {y } ]}$ \\
\hline Vélaires & S/[k] & & $\begin{array}{ll}\dot{\varepsilon} & \dot{\tau} \\
{[\dot{\mathbf{g}}]} & {[\chi]} \\
\end{array}$ & & & \\
\hline Uvulaire & ق[q] & & & & & \\
\hline Pharyngales & & & $\begin{array}{cc}\varepsilon & \tau \\
{[\mathrm{c}]} & {[\mathrm{h}]} \\
\end{array}$ & & & \\
\hline Glottales & $s /[2]$ & & $\therefore /[\mathbf{h}]$ & & & \\
\hline
\end{tabular}

Tableau 4

\section{F. Mécanisme de l'assimilation selon les traits pertinents}

Ce qui est important à noter, ce sont les modifications de nature que peut subir l'une des deux consonnes en contact. La plus faible est victime de la

22 -Sabio, (Fréderic), Phonétique et phonologie du français, P.17

ecrpe2015.unblog.fr/...phonologie-et-phonetique. 'pdf (consulté le 09/11/2013). 
plus forte. Pierre Fouché nous a énoncé des règles subtiles d'assimilation consonantique: ${ }^{23} \mathrm{Si}$ les deux consonnes en contact sont identiques, elles forment une géminée, c'est-à-dire que l'on prononce comme une seule consonne.

Ex. Un cheffameux (deux consonnes sourdes)

Ex. Un arabe blessé (deux consonnes sonores)

Ex. /qadd $\chi a 1 /$ قدْ دَخل (deux consonnes sonores)

a) $\mathrm{Si}$ les deux consonnes ont le même lieu et le même mode d'articulation, mais non la sonorité (l'une est voisée, l'autre est non voisée, ou inversement): la première devient voisée si la seconde est voisée, non voisée si la seconde est non voisée.

Ex. Une nappe blanche $(/ \mathrm{b} /$ sonorise $/ \mathrm{p} /)$

Ex. Une robe propre (/p/ assourdit $/ \mathrm{b} /)$

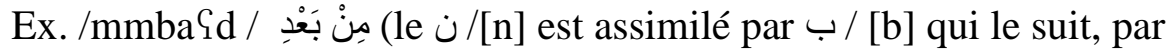
conséquent le ن ن est renversé à un phonème ayant le même point d'articulation que le ب / [b], c'est le / [m] car les deux sont des consonnes labiales.)

\subsection{La position des consonnes en contact}

\section{A. Les consonnes en contact dans la même syllabe}

Dans ce cas, les règles précédentes sur le rapport des forces consonantiques s'appliquent en générale très bien. Le phonème fort assimile le phonème faible. Martinet le montre avec cet exemple merveilleux: "autrefois, le mot

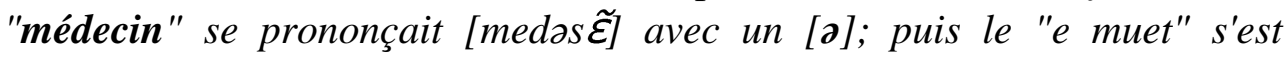
affaibli, on a dit /med्रs $\tilde{\boldsymbol{g}}$, et puis finalement /mets $\tilde{\boldsymbol{g}}$. C'est-à-dire qu'il y a anticipation graduelle de la surdité du /s/ affectant d'abord la voyelle /a/, puis le /d/, qui est passé à /d/d/, puis en se renforçant à $/ \mathrm{t} / »^{24} \mathrm{C}^{\prime} \mathrm{est}$ ainsi qu'on peut dire que le phénomène de l'assimilation est un phénomène de nature graduée plutôt que dichotomique.

En tombant le "e muet", «cheveu» se prononce / $\int \mathrm{f} \emptyset /$, les deux premières consonnes se trouvent dans la même syllabe. Dans cette fois, c'est la première consonne sourde $/ \int /$ qui assimile la deuxième sonore/v/.

Le même phénomène peut être réalisé dans les groupes des consonnes indissociables: consonne $+\mathrm{R}$ ou $\mathrm{L}$ (voir la figure1). Quand les deux consonnes sont sonores (comme dans bleu, drap, gros), elles gardent leur sonorité. Mais si la première consonne du groupe est non voisée, elle

23 - Fouché, (Pierre), Traité de prononciation française, Paris, C.KLINCKSIECK, 1959, PP.XLVI-XLIX

24 - Martinet, (André), (2001); Op.cit. p.115. 
"dévoise alors la sonore. Ex. très [t tre], clé [k/e] » ${ }^{25}$

Quant à l'arabe, cette règle est inapplicable car il est impossible que deux consonnes se rencontrent dans la même syllabe: " deux voyelles, deux consonnes ne peuvent jamais être en contact; l'hiatus y est impossible; et il est impossible que deux consonnes forment un chaînon, qu'il soit implosif ou explosif. Consonnes et voyelles y alternent ; d'où quatre séquences théoriquement possibles : $C V, C V C, V C$ et $V C V »{ }^{26}$ Il est à signaler que seules les deux premières séquences sont effectivement réalisées.

\section{B. Les deux consonnes en contact dans deux syllabes différentes}

Quand les deux consonnes en contact sont dans deux syllabes séparées, ce n'est pas la nature de la consonne qui compte mais sa position: les consonnes initiales de syllabe sont plus fortes et plus nettes (on les dit consonne explosives) que celles qui terminent la syllabe (on les dit consonne implosives). ${ }^{27}$ Dans absent, la division syllabique se fait entre $\langle\boldsymbol{b}$ et $\boldsymbol{s} \gg$. Bien que [b] soit une occlusive forte par nature, elle est en position faible, parce $q u$ 'en fin de syllabe. Par conséquent, le [s] initiale de syllabe assimile le [b] précédent et lui donne son caractère non voisé. Il devient sourd comme le $[s]$, donc[p], d'où la prononciation [apsãa]. ${ }^{28} \quad$ En arabe, le nūn as-

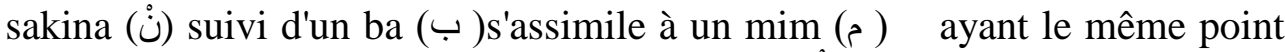
d'articulation du ba (ب) comme dans le mot (مَنْبر (منبر) prononcé (/mimar). Il est opportun de signaler que la modification se réalise au niveau phonique et non pas au niveau graphique, le nūn as-sakina (ن) se prononce comme un $\left([\mathrm{m}]_{\Gamma}\right)$ et il est toujours écrit $(\dot{\mathcal{U}})$.

\section{Les types de l'assimilation}

Les linguistes arabes modernes comme $\mathrm{Anis}^{29}$ et Chahine ${ }^{30}$ ont étudié en détail l'assimilation et l'ont classifiée en deux types essentiels: régressive et progressive. Ils signalent que «le phénomène de l'assimilation est plus

25 -Léon (Pierre), Phonétisme et prononciations du français, Paris, Nathan, 1994,p.71

26 - Roman (André), Remarques générales sur la phonologie de l'arabe classique, (ouvrage en français) in Revue de l'Occident musulman et de la Méditerranée, №15-16, 1973. PP.292-293

27 - Riegel (Martin) \& alii, Grammaire méthodique du français, Paris, QUADRIGE/PUF, $4^{\mathrm{e}}$ éd., 2009, p. 96.

28 - Léon (Monique) \& Léon (Pierre), prononciation du français, Paris, Nathan, 1997, P.60.

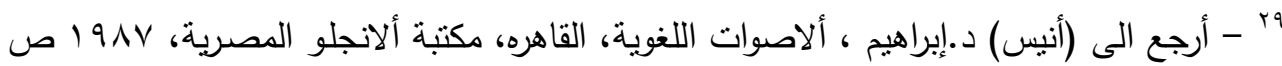

$$
\text { .r. T-IVA }
$$

• ץ - أرجع الى (ثاهين) د.عبد الصبور ،علم ألاصوات لبرتيل مالمبرج، دراسة وتعريب، القاهره،

$$
\text { مكتبة الثباب، } 1910
$$


fréquent dans la forme VIII $^{31}$ du verbe arabe (3iftacal إفتحل) où la première consonne de la racine (i.e. le faa (ف) du verbe racine) peut être د/ [d], ذ ذ j [z] ou l'un des sons emphatiques $/ \mathrm{d} /$ ض et

Nous pouvons classer les types d'assimilation selon trois paramètres:

- D'après la direction dans laquelle s'effectue le mouvement: régressive, progressive ou double;

- Selon leur caractère partiel ou total;

- Selon la distance: l'assimilation touche des sons contigus ou des sons à distance.

\subsection{Assimilation régressive, progressive ou double}

a) L'assimilation régressive ${ }^{33}$ ou anticipatrice se fait de droite à gauche, c'est-à-dire qu'un son assimile un autre son qui le précède. L'assimilation régressive est le type le plus fréquent d'assimilation en arabe et en français; elle peut principalement porter sur le voisement et la nasalité:

- assimilation de voisement (i.e. de sonorité) où le caractère voisé ou non voisé de la première est assimilé par la seconde.

Pour le français, un précurseur de ce type d'études est André Rigault qui, en 1967, a effectué une "étude acoustique et perceptuelle de l'assimilation consonantique de sonorité en français ${ }^{34}$. Cette étude acoustique a été faite sur des enregistrements de phrases contenant des occurrences d'assimilation ainsi que des occurrences non assimilées, permettant de les comparer. Cette analyse a mis en évidence une neutralisation du trait de sonorité (c'est à dire de voisement) dans des phrases telles que «guide savant» (où guide est prononcé comme [git]) par rapport à «fuite secrète» ou «guide zoulou», aussi bien entre les mots qu'à l'intérieur d'un même mot.

${ }^{31}$-La forme VIII (?iftaSal(إفتعل) a la particularité de réaliser un morphème " $t$ " non pas dans une position préfixale mais entre la première et la deuxième consonne de la racine. Cette forme a pour rôle d'indiquer le réflexif. Elle est considérée comme étant la forme réflexie de la forme I "fạal فعل". Toutefois, dans certains cas, ce rôle s'estompe et laisse apparaitre à la place une réciproque ou un passif. ARBAOUI (Nor elhouda), Les dix formes de l'arabe classique à l'interface syntaxe/phonologique, Thèse de Doctorat en Linguistique Université Paris 7 - Diderot, décembre 2010, p.60

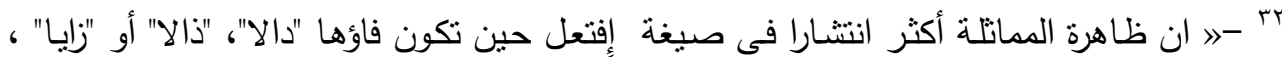

$$
\begin{aligned}
& \text { أو أحد اصوات ألاطباق/ ض/ ص/ ظ/ ط }
\end{aligned}
$$

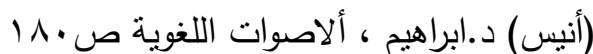

33 - Malmberg (Bertil), La Phonétique, Paris, PUF, 4éd. 1987, P.70.

34 - Rigault, A. (1967). L'assimilation consonantique de sonorité en français : Etude acoustique et perceptuelle. Papier présenté au $6^{\mathrm{e}}$ congrès international aux sciences de phonétiquePrague. 
L'assimilation de voisement se manifeste donc dans un seul mot ou à la jointure entre deux mots, et apparaît souvent en cas de chute du [ə]. Comme dans: paquebot [pagbo], médecin [mets $\tilde{\varepsilon}$ ] (voir figure 2) ${ }^{35}$ absence

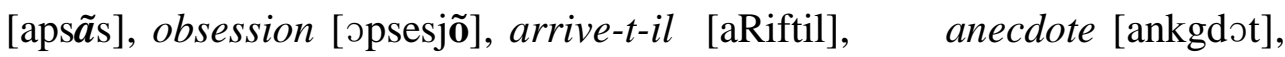
pas de problèmes [patpRoblem], coup de couteau [kutkuto]

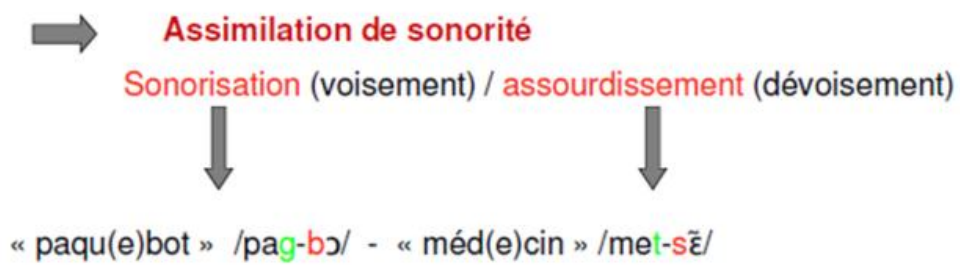

Figure 2

En arabe populaire, le sin (w) non voisé suivi d'un țā ( ط ) emphatique s'assimile à un sād (ș̣ ص (مسطره) comme dans le mot prononcé (مصطره /mașțarah). Même en arabe classique, comme dans le verbe

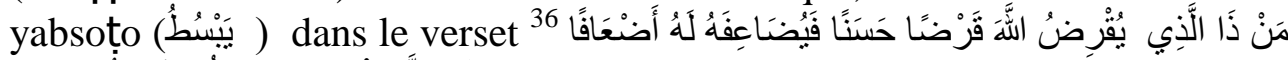

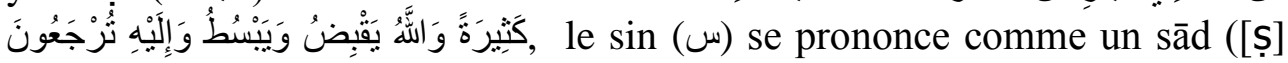
ص ) sous l'influence de l'emphatique țā ( b)

- assimilation de nasalité: c'est le second type de l'assimilation régressive le plus fréquemment attesté en français : lorsqu'une occlusive est précédée d' une voyelle nasale et suivie d' un son de prononciation nasale, elle tend ellemême à se nasaliser.

En parlant rapidement, on prononce $[\mathrm{p} \tilde{a} \mathrm{n} \tilde{\boldsymbol{a}}]$ pour pendant, un $[\boldsymbol{n}]$ à la place d'un $[\boldsymbol{d}] \quad$.Ce sont les deux voyelles nasales $[\tilde{a}]$ qui favorisent le remplacement de la consonne occlusive voisée [d] par la consonne nasale sonore correspondante[n].

La consonne occlusive et la nasale qui la remplace doivent garder le même lieu d'articulation, soit :

- dental : $\mathbf{t} / \mathbf{d} \rightarrow \mathbf{n}$, c'est le cas le plus fréquent, comme dans: pendant

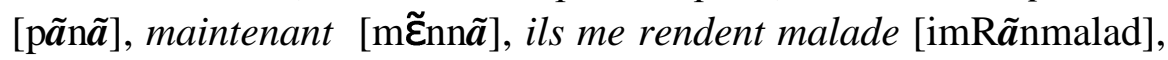
grandement $[\mathrm{gR} \tilde{\boldsymbol{a}} \mathrm{nm} \tilde{\boldsymbol{a}}]$

Signalons que la prononciation nasalisée de «pendant » et «maintenant » semblent avoir été adoptée par la plupart des français.

35 -Christelle Dodane, Phonétique Française, asl.univ-montp3.fr/phonetique/cdodane/.../TD4-TD6-.

36 - Le Saint Coran, Sourate Albaqarah, verset 245. 


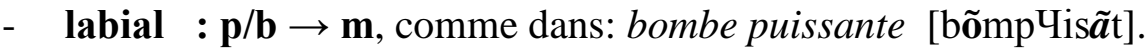

En arabe, l'assimilation de nasalité est plus fréquente à la récitation du Coran sous forme de ce que les savants de Tajwid appellent" règles liées au mime et Noune saakine et Tanwine":

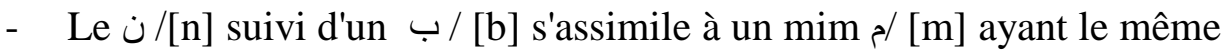
point d'articulation du ب/[b],(car les deux sont des consonnes

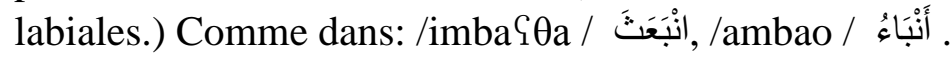

- En outre, dans un certain nombre des mots outils (comme أنْ، إنَّ ،عنَ ، منِ le nūn final s'assimile à un mīm, ce qui donne les prononciations suivantes :

$-\mathbf{N}+\mathbf{M} \rightarrow \mathbf{M M}$

Ex: $\bar{a} n+m a \bar{a} \rightarrow$ ammā

Ex: $\operatorname{mī}+\operatorname{ma} \rightarrow$ mimma

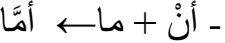

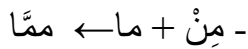

Ex: \an + mā $\rightarrow$ \ammā

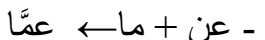

le nūn peut aussi s'assimiler à un lām comme dans:

$-\mathbf{N}+\mathbf{L} \rightarrow \mathbf{L L}$

Ex: Pan + lā $\rightarrow$ ?allā

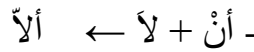

- إنَّ

Ex: Pin + lā $\rightarrow$ ?illā

Il est à remarquer que ces séquences sont graphiées comme un seul mot, produisant les formes إلاّ, ألاّ ممَّا، عِمَّا, أمَّا .

b) L'assimilation est dite progressive lorsque, dans une suite de deux consonnes, la première assimile la deuxième. En prononçant «cheveu» avec l'élision du son [ə] comme [ [fø], la première consonne [ [] assimile la suivante[v]. En français, on a une assimilation progressive pour [R] ou [1] chaque fois qu'elles apparaissent après une consonne sourde, telles [f], [k], [t] ou [p] dans des mots comme offres, boucle, neutre, peuple, etc.

L'assimilation progressive est plus fréquente en arabe qu'en français. Dans

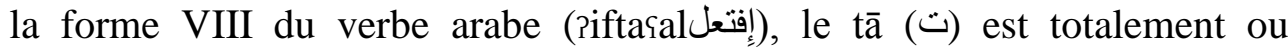
partiellement assimilé par:

- les phonèmes: $[\mathrm{d} / \mathrm{د}],[ð / \mathrm{J}]$ et $[\mathrm{z} / j]$ comme dans:

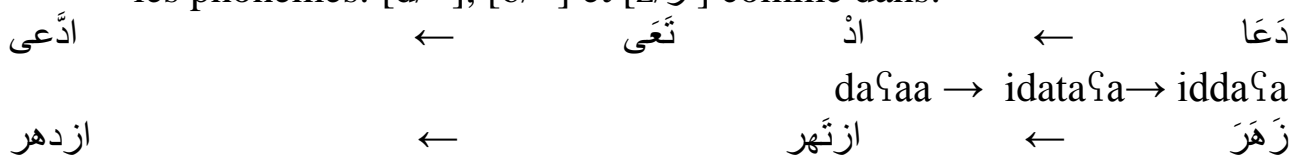

Piðtakara $\rightarrow$ Piðdakara $\rightarrow$ Pidakara $\quad$ أَكَر

ðakara $\rightarrow$

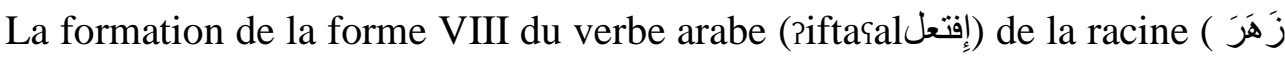

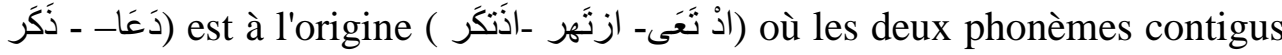


sont différents: le premier $([\mathrm{d} / \mathrm{\lrcorner}],[\mathrm{d} / \mathrm{\lrcorner}]$ ou $[\mathrm{z} / \mathrm{j}])$ est voisé; le second $([\mathrm{t} / ت])$ est non voisé. Les voisés $[\mathrm{d} / \mathrm{د}],[\mathrm{d} / \mathrm{j}]$ et $[\mathrm{z} / j]$ assimilent

- Dans le premier exemple, le tā (ت) infixé est assimilé par le le dāl (د) en se transformant en $[\mathrm{d} / \mathrm{s}]$. Les deux s'amalgament et se prononcent en $[\mathrm{d} / \mathrm{د}]$ géminé [iddasa/ ادَّعى] ].

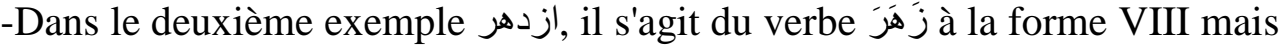
le $[\mathrm{t} / ت$ ] infixé de la VIII s'est transformé en $[\mathrm{d} / \mathrm{L}$ ] à cause du $[\mathrm{z} / j]$ qui l'a sonorisé. Le $[\mathrm{d} / \mathrm{s}]$ est parfaitement convenu car il a le même point d'articulation que $[\mathrm{t} / ت$ ] (occlusive dentale) et avec le $[\mathrm{z} / j]$ car les deux sont sonores.

- Quant au troisième exemple, il présente deux étapes de l'assimilation: au premier temps le $[\mathrm{t} / \Xi$ ] infixé est assimilé par le [ð/ذ ] en se transformant en

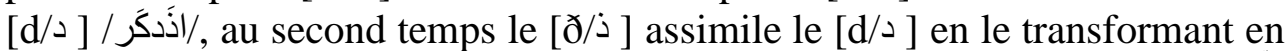

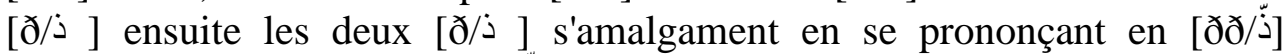
géminé. Le même mot اذذّكر/ اذكر/ est subie parfois sous l'influence de l'assimilation régressive: /ادّكر/la se prononce/, c'est la forme la plus employé dans la pratique, on dit qu'il y a dans ce cas une assimilation partielle regressive où le premier $[\partial / ذ]$ est assimilé par le deuxième $[\mathrm{d} / \mathrm{\Delta}]$. Les deux s'amalgament en [دّ] géminé.

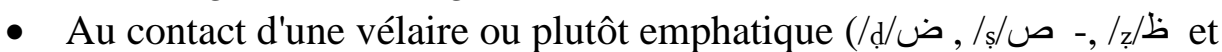

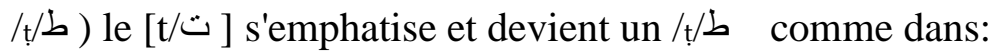

șafā $\rightarrow$ iistafā $\rightarrow$ iisțafā

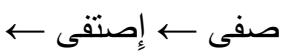

إصطفى

țalasa $\rightarrow$ Rițtalasa $\rightarrow$ ?ițtalasa

طلع ๘ إطتلع

طّلع

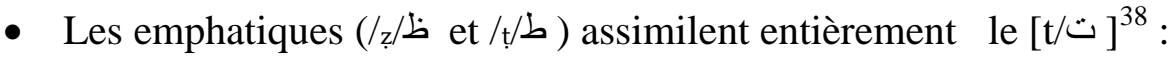

țarada $\rightarrow$ Pițtarada $\rightarrow$ Piț̣arada

طرد - م إطترد

إطِّرد

zalama $\rightarrow$ Piztalama $\rightarrow$ Pizzzalama

ظُّلم

rr "- في ادّكر صار تطور آخر : فقنى الصوت الثانى فى ألاول ونطق بهما صوتا واحدا كألاول،

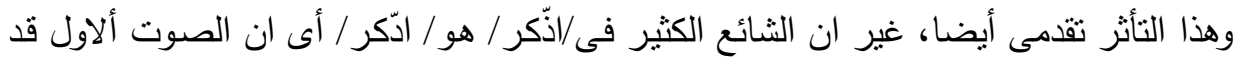

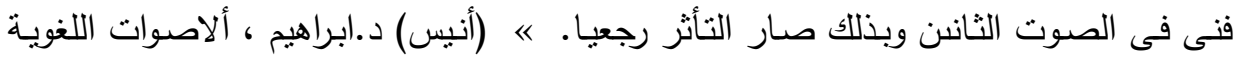

$|\wedge|-1 \Lambda \cdot ص$

38 - Blachère (Régis) et Gaudefroy-Demombynes (M.), Grammaire de l'arabe classique, Maisonnette-Larose, Paris, 1994, P.66. 
c) L'assimilation peut aussi s'exercer tout à la fois régressive et progressive. Pour les termes en -isme, de façon générale deux prononciations sont attestées [ism/izm] selon les régions: «Les Français du Nord disent communisme, avec un [m] assourdi par le [s] qui se trouve dans la même syllabe. Les Français du Midi prononcent [komynizma]. À cause du $\boldsymbol{E}$ final prononcé, le $[\mathrm{m}]$ devenu initial de syllabe assimile le [s] précédent, qui devient [z].» ${ }^{39}$ Ainsi, dans une séquence telle que «je crois», si le [ə] n'est pas prononcé, l'assimilation «s'exerce de deux côtés [ $\left.\int k \hat{w} a\right]$ puisque le [k] assourdit à la fois la consonne qui le précède et celle qui le suit ${ }^{40}$ car elles sont dans la même syllabe.

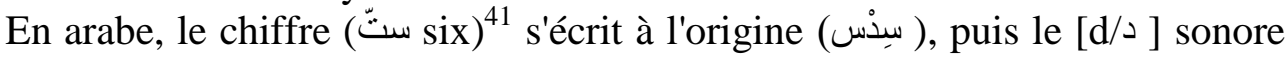
est assimilé par le [s/w] sourd, il est substitué par son équivalent sourd le [t/ ], par conséquent le chiffre est devenu (ستس ), ensuite le [t/ت] assimile le [s/w en $[t / ت]$; finalement une gémination est effectuée, le chiffre est devenu (ستّ (س) Ainsi le chiffre est subi au premier temps sous l'influence de l'assimilation régressive, au deuxième sous l'influence de l'assimilation progressive.

d) L'assimilation peut être double ${ }^{42}$ : dans ce type, le son est assimilé par les deux sons qui l'entourent. Comme dans le cas d'une consonne intervocalique : «Les consonnes en position intervocalique sont susceptibles de subir [...] des assimilations de sonorité [...]. Dans le mot second, le [k] entre deux voyelles est sonorisé à la fois par assimilation régressive et progressive.» ${ }^{43}$ Ainsi dans l'exemple de «maintenant» [mẽtnã], le phonème /t/ peut être nasalisé, étant entouré par une voyelle et une consonne nasales, ce qui

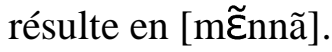

39 -Léon (Monique) \& Léon (Pierre), prononciation du français, Op.cit.P.61.

40 -Léon (Pierre), Phonétisme et prononciations du français, Op.cit.P.72.

ו؛ -» العدد" ستّ" فى العربية، ألاصل فيه " سِذس " تأثثرت الدال المجهورة بالسين المهوسة فقلبت

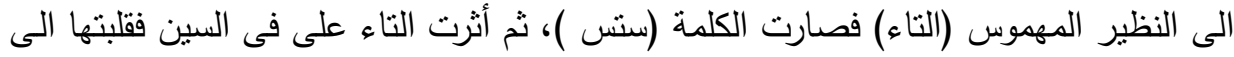

تاء، ثم حصل ادغام فصسارت" ستّ" وهنا مرت بمرحلتين فى ألاولى تأثثر مدبر وفى الثانية

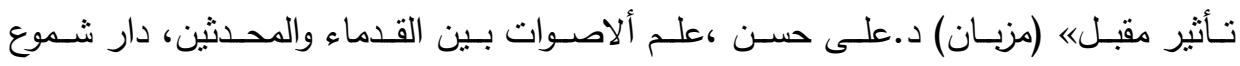

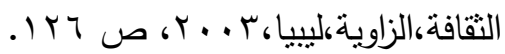

42 - Malmberg, (Bertil), Op.cit. p.70.

43 -Argot-Dutard (Françoise.) : Éléments de phonétique appliquée, Paris, A. Colin/ Masson, 1996, P. 60. 
En arabe, dans la forme VIII, si la deuxième lettre de la racine, et non la première, est emphatique, l'assimilation s'effectue alors le plus souvent phonétiquement, et non graphiquement. Le radical (نَظًر ) à la forme s'écrit

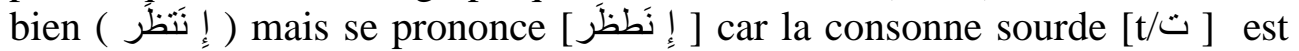
suivie par l'emphatique[ ط

\subsection{Assimilations partielles ou totales}

L'assimilation est partielle quand le son transformé par assimilation ne va pas jusqu'à se confondre entièrement avec le son contigu. Dans les deux exemples, «Chapdelaine [pd]; bec de lièvre [ $k d]$, les [p] et [k], en fin de syllabe, sont en position implosive (faible); c'est pourquoi ils subissent l'influence de [d], plus fort parce qu'explosif (à l'initial de syllabe); celui-ci leur communique une partie de sa sonorité: c'est une assimilation de sonorité régressive partielle. ${ }^{44} \mathrm{En}$ arabe, l'assimilation est partielle lorsque les deux lettres n'ont pas le même point d'articulation, mais elles ressemblent phonétiquement de telle manière qu'on arrive à les prononcer sans difficulté comme dans la forme VIII [إصنتَدم] qui se prononce [إصطَّم] à cause de l'emphatique $/$ ص ص Les deux lettres peuvent avoir le même point

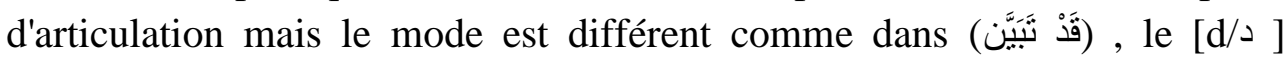
sonore est assimilé par le $[\mathrm{t} / \bullet$ ] sourd en perdant son voisement et prononçant comme le non voisé $[\mathrm{t} / \bullet$ ].

Il y a toutefois des cas où l'assimilation peut être totale, c'est-à-dire avoir pour conséquence une adaptation intégrale au son contigu. En français moderne, dans un parler rapide spontané, les assimilations sont totales. Ainsi dans l'assimilation de sonorité ou de sourdité, /b/ devient $/ \mathrm{p} /$ et $/ \mathrm{p} /$ devient /b/ comme dans: absent [apsã], obscur [opskyr], une nappe blanche [ynnabblã ]], une robe propre [ynroppropr].

En arabe, l'assimilation totale se manifeste principalement avec l'article défini أَ /Ral/ qui est assimilé par la première consonne solaire du nom qui le suit comme dans /at' tūt/النّّتو, /an' nās/ النّاس/.

L'article [ أَّal] se traite de deux façons :

Quand l'article n'est précédé d'aucun mot : il sera alors assimilé devant le mot qui commence par une lettre solaire. Avec ces lettres, l'article va donc subir une assimilation, c'est-à-dire l'article sera prononcé [a / I], et la lettre commençant le mot sera doublée. On insistera sur sa prononciation et à l'écrit elle porte une chadda الرَّحمَنُ الرحمَ par /ar-rahman/ ;

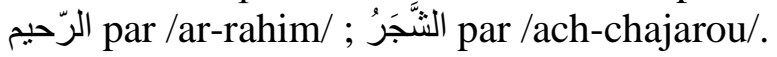

44 - Carton (Fernand), Op.cit. P.83. 
Quand l'article est précédé d'un mot: il ne se prononce plus du tout. On fait directement la liaison entre le mot qui le précède et le mot qu'il définit et cela rend la prononciation beaucoup plus facile. Sans prononcer l'article, on fait une liaison directe entre le premier mot et la lettre solaire du deuxième mot en doublant celle-ci, c'est-à-dire en insistant sur cette lettre, comme dans: مَّنَ الرَّخْمَن se prononce mmina-r-rahmani.

\subsection{L'assimilations à distance}

Au contraire de l'assimilation qui touche les sons contigus, il peut arriver que l'assimilation influence des sons à distance. Ce phénomène peut se produire non seulement pour les consonnes (la dilation) mais aussi pour les voyelles (harmonisation vocalique).

a) La dilation: "C'est le transfert de traits distinctifs d'un phonème sur d'autres phonèmes non contigus, par anticipation ou par inertie.» ${ }^{45}$ Certains français disent pour «chasseur» [ $\left.\int \mathrm{a} \int œ r\right]$ au lieu de [ $\left.\int \mathrm{asœr}\right]$; cette prononciation est le résultat de «l'influence de la chuintante initiale [S] sur la sifflante [s] qui est transformée elle aussi en chuintante [S].» ${ }^{46}$

Le changement par dilation peut être total, comme le mot de l'ancien français «cerchier [tsert Jjer]» qui devient «chercher $\left[\int \varepsilon r \int e\right] »{ }^{47}$ en français moderne.

Il convient de mentionner que l'assimilation à distance par dilation est une formule peu fréquente en français.

\section{b) L'harmonisation vocalique}

Il s'agit du transfert de traits distinctifs d'un phonème vocalique sur un autre non contigu. Dans une comparaison par spectrogramme du timbre de l'article dans «les lits» et dans «les baies», Carton ${ }^{48}$ a remarqué que la voyelle du premier «les» est plus fermé que celle du second. Le timbre de la voyelle inaccentuée $E$ est influencé par celui de la voyelle accentuée: [i] est plus fermé que $[\varepsilon]$.

L'adverbe «aujourd'hui» [ozu:RdYi] est très fréquemment prononcé [o3 ๑:RdЧi]: le [u] est attiré par le [o] initial et s'ouvre par la loi de position (en syllabe fermée, la voyelle a tendance à s'ouvrir). En franco-québécois et chez les arabophones, l'adverbe «beaucoup» ${ }^{49}$ se prononce [buku] au lieu de [boku]; le [u] final a exercé une influence fermante sur le [o] de la syllabe

45 - Carton (Fernand), Op.cit.p.137.

46 - M.-c.Thomas (Jacqueline), Bouquiaux(Luc), France Heiss (Cloarec-); Initiation à la phonétique, Phonétique articulatoire et phonétique distinctive, Paris, PUF, 1976.P.131.

47 - Carton (Fernand), Op.cit. p.138.

48 - Ibid.p.87.

49 -andre.thibault.pagesperso-orange.fr//phonologie semaine 3 (consulté le20-11-2013). 
précédente, entraînant sa fermeture d'un degré d'aperture, ce qui a eu pour effet de transformer ce $[\mathrm{o}]$ en $[\mathrm{u}]$.

Il est important de signaler que l'assimilation vocalique se produit essentiellement avec la voyelle E ouvert, en syllabe ouverte, en position inaccentuée, qui devient $\mathrm{E}$ fermé sous l'influence d'une des trois voyelles fermées: [i], [e], [y]. Comme dans ${ }^{50}$ : bête [bst], bêtise [betiz], aime [cm], aimé [eme], tu sais [tyse], sais-tu [sety]. On estime que dans le cas des voyelles, " leurs variantes conditionnées les plus intéressantes ne concernent pas leur timbre mais leur longueur $\gg .{ }^{51}$

C'est un phénomène d'assimilation régressive à distance : le $[\varepsilon]$ ouvert, en syllabe ouverte, suivi d'une voyelle fermée accentuée, se ferme: j'aime [3єm], vous aimez [3eme].

En arabe, la préposition bī (ب) a normalement la voyelle [i], celle-ci

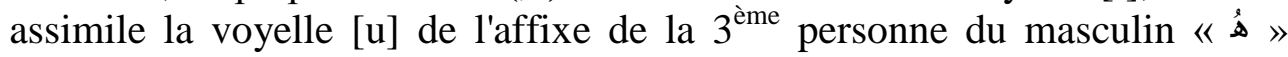
agglutiné à la préposition en la transformant en «i» pour donner (بد ). La préposition (ل) ayant la voyelle [i] devient (J) si celle-là est agglutinée à

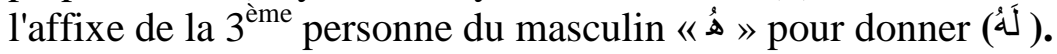

\section{Dissimilation}

La dissimilation est l'inverse de l'assimilation, c'est tout changement phonétique qui a pour but d'accentuer ou de créer une différence entre deux sons voisins. D'un autre terme, c'est "la modification d'un trait phonétique d'une consonne qui se trouve en contiguïté avec une consonne de même nature» ${ }^{52}$. Ce phénomène, à l'opposition de l'assimilation, n'est fréquent ni en arabe ni en français.

Pour Malmberg ${ }^{53}$, il s'agit le plus souvent d'éviter une répétition gênante entre deux phonèmes identiques. C'est de cette façon qu'il faut expliquer le français populaire colidor pour corridor ou le français moderne couloir d'un plus ancien couroir. La dissimilation peut également affecter les voyelles, comme dans ${ }^{54}$ : lat. divisa $>$ Fr. devise $(\mathrm{i}-\mathrm{i}>\mathrm{e}-\mathrm{i})$.

En arabe, la dissimilation a pour but de modifier l'un des deux phonèmes identiques contigus à l'intérieur d'un même mot ou entre deux mots. Le phonème modifié peut être "s soit l'un des sons de Madd( prolongement): le (I) Alif [a],(و) le waw [w] et le (ى) yaa [y]; soit l'un des phonèmes qui

\footnotetext{
50 - Léon (Monique) \& Léon (Pierre), prononciation du français, Op.cit.P.63.

${ }^{51}$-Riegel (Martin) \& alii, Op.cit., p.96

52 - Choi-Jonin (Injoo) \& Delhy (Corinne), op.cit. p.59

53 - Malmberg, (Bertil), Op.cit. p.72.

54 - Carton (Fernand), Op.cit. P.137
} 
n'exigent pas d'effort à la prononciation comme : le lām (J), le raa (J), le noun (ن) ou le mim (^) »55

Considérons ces deux exemples:

din-nar $\rightarrow$ di-nar

qirrat $\rightarrow$ qi-rat

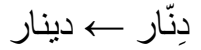

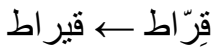

Chacun de ces deux exemples comporte deux phonèmes identiques : le noun géminé au premier et le raa géminé au deuxième. En vue de faciliter la prononciation, le premier noun(ن) et le premier raa $(J)$ ont été substitués par le yaa (ى). Le pluriel de ces deux mots prouve que cette substitution est à la fois phonologiquement et morphologiquement correcte:

di-nar $\rightarrow$ da-na-nir

دينار ك دنانير

qi-rat $\rightarrow$ qa-ra-rit

قير اط ـ ثراريط دنانير

Dans ( إنَّ + ما ), le second Noun se renverse en Mim qui s'agglutine au Mim initial faisant un Mim géminé, on prononce les deux monèmes amalgamés de cette façon: /inamma/إنمَّ/

\section{Conclusion}

Dans cette étude, nous avons abordé l'assimilation, ses contraintes et ses aspects comme une variation phonologique observée dans les deux langues: le français et l'arabe. L'analyse de ce phénomène nous a révélé quelques traits essentiels communs entre les deux langues en question et qui sont censés être universels. Nous les passons ainsi en revue:

- Le mécanisme de l'assimilation est conditionné par une connaissance phonologique spécifique au système vocalique des langues en question: traits pertinents des phonèmes et leur contact dans un contexte phonologique quelconque.

- L'assimilation peut se produire entre deux mots ou au sein d'un mot, ce qui change généralement la structure phonique interne : comme dans le cas du monème "médecin".

- L'assimilation est de nature graduée: il existe des degrés de transformation d'un son en un autre (partielle ou totale): en cas d'assimilation totale, le phonème assimilé est influencé par le

0 - >فى العربية تهدف ظاهرة الخالفة الى تغيير احد الصوتين المتماتلين الى صوت آخر كأصوات المد: ألالف والولو والياء أو أحدألاصوات الثبيهة بها وهى ألاصوات المتوسطة اوالمائعة ممثلة

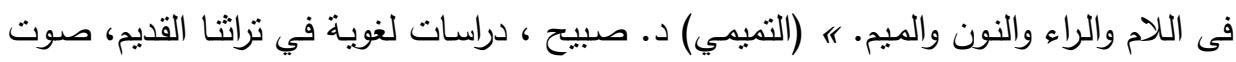

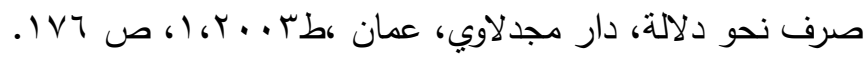


contexte droit, mais lorsqu'il s'agit d'une assimilation partielle, les traits pertinents des phonèmes influencent le processus de l'assimilation.

- Il s'est avéré que les sons non voisés se voisent plus facilement et plus complètement que les sons voisés.

- L'assimilation régressive est le type le plus fréquent surtout au niveau du voisement et de la nasalité.

- L'assimilation à distance représentée par la dilation (portant sur les consonnes) et par l'harmonisation vocalique (portant sur les voyelles) touche les sons non contigus.

- La dissimilation, à l'opposition de l'assimilation, n'est pas fréquente dans les deux langues.

En outre, le traitement contrastif de l'assimilation entre les deux langues, nous révèle quelques aspects propres à chacune d'elles:

- En français, l'assimilation s'effectue comme un résultat de la rencontre réelle de deux consonnes, de la rencontre produite de la chute "e" muet ou dans les groupes des consonnes indissociables à l'intérieur d'un mot (e.g. bleu) ou entre deux mots ( une nappe blanche). En arabe, l'application de l'assimilation de cette façon est impossible car deux consonnes ne peuvent jamais être en contact: consonnes et voyelles s'alternent obligatoirement $(\mathrm{CV}, \mathrm{CVC}, \mathrm{VC}$ et VCV).

- À l'opposé du français, l'assimilation progressive est fréquente en arabe surtout dans la forme VIII du verbe.

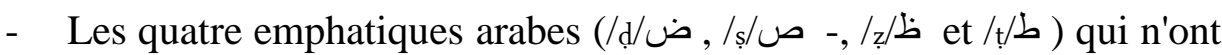
pas d'équivalent en français, assimilent généralement l'infixe "t" de la forme VIII du verbe malgré la différence du point d'articulation.

- En français moderne, l'assimilation totale de sonorité ou de surdité paraît au cas d'un parler rapide spontané. En arabe moderne et classique, l'assimilation est toujours totale avec l'article défini أَّet al/ qui est assimilé par la première consonne solaire du nom qui le suit.

Pour conclure, il s'avère que la compréhension de ce que dit quelqu'un qui parle sa langue maternelle ou bien une langue seconde n'est pas généralement une question simple. La reconnaissance des mots dans le flux phonologique continu exige une connaissance profonde non seulement du système phonétique de la langue en question mais aussi de la variabilité phonologique qui caractérise le langage parlé comme dans le cas de l'assimilation. 


\section{Bibliographie}

\section{I- REFERENCES EN LANGUE FRANÇAISE}

\section{- OUVRAGES THÉORIQUES}

Argot-Dutard ,F.(1996) : Éléments de phonétique appliquée, Paris, A. Colin/ Masson.

Blachère, $\mathbf{R}$. et Gaudefroy-Demombynes ,M.(1994), Grammaire de l'arabe classique, Maisonnette-Larose, Paris.

Builles,J.M. (1998), Manuel de linguistique descriptive, Le point de vue fonctionnaliste, Paris, Nathan.

Carton,F (1997), Introduction à la phonétique française, Paris, Dunod,.

Choi-Jonin, J. \& Delhy,C. (1998), Introduction à la méthodologie en linguistique, Paris, Presses universitaires de Strasbourg.

DARCY,I (2006), Assimilation phonologique et reconnaissance des mots, éd. Peter Lang SA,Bern,Berlin, Bruxelles,Frankfurt am Main,New York, Wien.

Dell,F. (1985), Les règles et les sons. Introduction à la phonologie générative, Paris, Hermann.

Derivery,N. (1997), La phonétique du français, Paris, Seuil, .

Fouché,P. (1959), Traité de prononciation française, Paris, C.KLINCKSIECK,.

Grammont,M. (1933). Traité de phonétique. Paris : Delagrave.

Gumbretière,E. (1994), Phonétique et enseignement de l'oral, Paris, Didier/Hatier.

Léon,p. (1994), Phonétisme et prononciations du français, Paris, Nathan.

Léon,M \& Léon,P. (1997), prononciation du français, Paris, Nathan,.

M.-c. Thomas,J. \& alii,(1976); Initiation à la phonétique, Phonétique articulatoire et phonétique distinctive, Paris, PUF.

Malmberg (Bertil), La Phonétique, Paris, PUF, $4^{\mathrm{e}}$ éd. 1987 Martinet,A. (1956), La description phonologique, avec application au parler franco-provençal d'Haute ville (Savoie), Genève, Droz et Paris, Mainard, .

ID,( 1965), linguistique synchronique, Paris, P.U.F,.

ID, (1971), De l'assimilation de sonorité en français, Form and substance, Mélanges Eli Fischer-Torgensen, Copenhague,.

ID,( 1974), Le français sans fard, Paris, P.U.F.

ID, (1998) Éléments de linguistique générale, Paris, A. colin $4^{\mathrm{e}}$ éd.

Mounin (Georges), Clef pour la linguistique, Paris, éd. Seghers, 1971.

Riegel,M \& alii(2009), Grammaire méthodique $d u$ français, Paris, QUADRIGE/PUF, $4^{\mathrm{e}}$ éd. 
Sabio, (Fréderic), Phonétique et phonologie du français, P.17

ecrpe2015.unblog.fr/...phonologie-et-phonetique. 'pdf (consulté le 09/11/2013).

Troubetzkoy,N.( 1970), Principes de phonologie, Klincksiek, Paris,.

Walter,H. (1977.), La phonologie du français, Paris, PUF, 4e éd.

\section{- THESES ET OUVRAGES INÉDITS}

- Albert, A. (2007), Éléments pour une description dynamique du système vocalique de l'arabe, Università di Cagliari.in Studi di Glottodidttica,4,http://www. Glottodidttica.net/

-Arbaoui,N. (2010), Les dix formes de l'arabe classique à l'interface syntaxe/phonologique, Thèse de Doctorat en Linguistique Université Paris 7 - Diderot, décembre.

-Boukadida, N. (2008), Connaissances phonologiques et morphologiques dérivationnelles et apprentissage de la lecture en arabe, (étude longitudinale) - Université Européenne de Bretagne, Université Rennes2 Université de Tunis I, Ecole doctorale - Humanité et Sciences de l'Homme, Faculté des Sciences Humaines et Sociales-Tunis,

-DJOUDI, M (1991), Contribution à l'étude et à la reconnaissance automatique de la parole en Arabe standard, thèse de Doctorat de l'université de Nancy 1, Novembre.

-Kabbour,K. 1988), L'Assimilation en arabe classique: essai d'une analyse autosegmentale, A.N.R.T,.

- Rigault, A. (1967). L'assimilation consonantique de sonorité en français : Etude acoustique et perceptuelle. Papier présenté au $6^{\mathrm{e}}$ congrès international aux sciences de phonétique .Prague.

\section{- ARTICLES}

Dodane, Ch., Phonétique Française, asl.univ-montp3.fr/phonetique/cdodane/.../TD4-TD6-.

Martinet, A. (2001), La phonologie, in La linguistique, Les introuvables d'André Martinet, volume36, 2001-1/2, Paris, PUF, , P.262.

Rigault, (A.) (1967). L'assimilation consonantique de sonorité en français : Etude acoustique et perceptuelle. Paper presented at the Proceedings of the 6th International Congress of Phonetic Sciences, Prague.

Roman, A. (1973), Remarques générales sur la phonologie de l'arabe classique, (ouvrage en français) in Revue de l'Occident musulman et de la Méditerranée, $\mathrm{N}^{\circ} 15-16$,

\section{- DICTIONNAIRES CONSULTÉS}

Dictionnaire Hachette encyclopédique, Paris, Hachette, 2005.

Dubois (Jean) et alii, Grand Dictionnaire, Linguistique \& Sciences du Langage, Paris, Larousse, 2007. 
Le Petit Robert(1986), Dictionnaire de la langue française 1, Paris, Le Robert.

Maingueneau, D. (1996), Les termes clés de l'analyse du discours, éd. Paris, Seuil,.

Mounin, G. (1995), Dictionnaire de la linguistique, Paris, PUF,.

Oswald Ducrot \& Tzvetan Todorov, Dictionnaire Encyclopédique des sciences du langage, Pais, Seuil, 1972.

webographie

theses.sciences@scd.uhp-nancy.fr.

andre.thibault.pagesperso-orange.fr//phonologie semaine 3 (consulté le20-

11-213).

asl.univ-montp3.fr/phonetique/cdodane/.../TD4-TD6-.

Résumé des Règles du tadjwîd أحكام تلاوة القرآن الكريم برواية حفص،/P.53

www.islamhouse.com/d/files/fr/ih.../fr-Islamhouse-regles tajwid.pdf

Ahmed (Mourchad) TOCHA, J'apprends à lire l'Arabe du Qu'ARN (Coran), 3ème Version, 2013p.15

ekladata.com/LIQYnt55Le0Fxit6_qTRt6QH94E.pdf

ecrpe2015.unblog.fr/...phonologie-et-phonetique. 'pdf

http://ojs.cimedoc.uniba.it/index.php/glottodidattica/article/download/28/ 


\section{II- REFERENCES EN LANGUE ARABE}

- (التميمي) د. صبيح ، دراسات لغوية في تراثنا القديم، صوت صرف نحو دلالة،

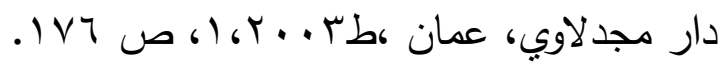

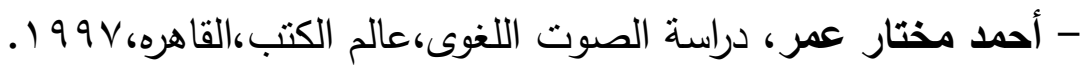

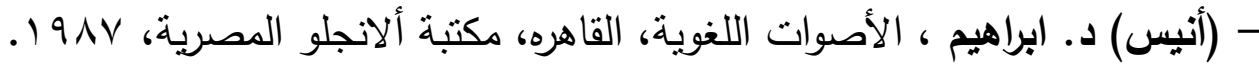

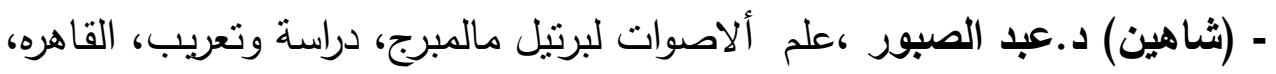

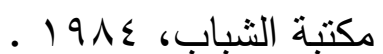

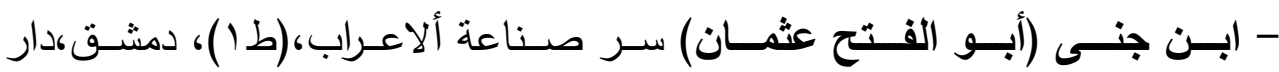
القلم،1910.

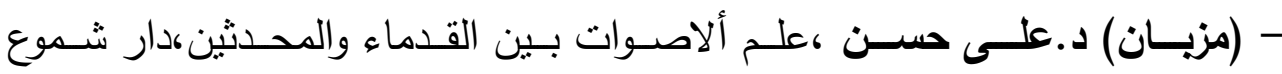

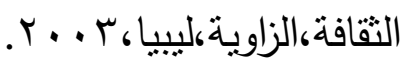

- سييويه،|لكتاب،تحقيق عبد السـلام محمد هارون،مكتبة الخانكى ، القاهرهط.ب،

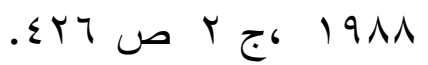

